\title{
Lucentis ${ }^{\circledR}$ using Visudyne ${ }^{\circledR}$ study: determining
} the threshold-dose fluence of verteporfin photodynamic therapy combined with intravitreal ranibizumab for exudative macular degeneration

\author{
This article was published in the following Dove Press journal: \\ Clinical Ophthalmology \\ 17 September 2010 \\ Number of times this article has been viewed
}

\author{
Eric Chen \\ David M Brown \\ Tien P Wong \\ Matthew S Benz \\ Eric Kegley \\ Joel Cox \\ Richard H Fish \\ Rosa Y Kim \\ Retina Consultants of Houston, \\ Texas, USA
}

Correspondence: Eric Chen Retina Consultants of Houston, 6560 Fannin Street, Suite 750, Houston, TX 77030, USA

Tel +I 7I3 5243434

Fax +I 7135243220

Email ecmd@houstonretina.com
Purpose: Combination verteporfin photodynamic therapy (vPDT) and antivascular endothelial growth factor (anti-VEGF) therapy may decrease the need for injections while maintaining visual acuity in exudative age-related macular degeneration. This pilot study was designed to determine the threshold fluence dose of vPDT (the dose required to demonstrate an effect on choroidal perfusion) combined with ranibizumab.

Methods: Seven patients were randomized to sham vPDT (two patients), 20\% fluence vPDT (two patients), or 40\% fluence vPDT (three patients) in combination with three-monthly intravitreal $0.5 \mathrm{mg}$ ranibizumab injections. Intravitreal ranibizumab was reinjected if disease activity was seen on fluorescein angiography, optical coherence tomography, or clinical examination. Indocyanine green-determined choroidal hypoperfusion was graded in a masked fashion.

Results: Patients with 20\% vPDT had mild hypoperfusion defects at seven days that resolved by week 4 (threshold dose); patients with $40 \%$ fluence vPDT had marked hypoperfusion at seven days that persisted as long as 12 months. Recruitment was stopped after limited efficacy was observed. One patient with $20 \%$ fluence vPDT lost 19 letters at one year; no other patient lost or gained $>10$ letters. Central retinal thickness decreased in six of seven patients, but ranibizumab injections did not decrease.

Conclusion: This pilot study shows that the threshold fluence dose of vPDT (when combined with ranibizumab) is approximately $20 \%$ standard fluence, and that mild and transient choroidal hypoperfusion can occur. Forty percent fluence vPDT causes a more prolonged and striking hypoperfusion. Despite hypoperfusion, no decrease in visual acuity or injections required was noted, suggesting that even higher fluence levels of vPDT may be necessary to decrease the number of anti-VEGF injections.

Keywords: neovascular age-related macular degeneration, choroidal hypoperfusion, threshold dose, verteporfin photodynamic therapy

\section{Introduction}

Age-related macular degeneration (AMD) is the most common cause of legal blindness in the elderly in the Western world, and the prevalence of AMD is expected to increase by more than $50 \%$ by the year $2020 .^{1}$ There are two forms of AMD, ie, nonexudative and exudative. The exudative form, defined by the growth of a choroidal neovascular membrane (CNVM) and a subsequent increase in vascular permeability, is present in $20 \%$ of patients, and accounts for $90 \%$ of severe vision loss in patients with AMD. ${ }^{2}$

Verteporfin (Visudyne ${ }^{\circledR}$; QLT Ophthalmics, Inc., Menlo Park, CA, USA) photodynamic therapy (vPDT) is a therapy introduced in the late 1990s as a treatment for neovascular 
AMD. vPDT causes selective vascular occlusion of CNVM vessels, and the initial fluence levels and dose-escalation response of verteporfin PDT were determined in animal models. $^{3-5}$ Because the initial dose response curves of vPDT were completed in primates, the actual threshold and toxic doses of vPDT in humans were never accurately determined (Andrew Strong, PhD, QLT Pharmaceuticals, personal communication, 2008). This fluence dose was then used in the pivotal Treatment of age-related macular degeneration with photodynamic therapy and Verteporfin in photodynamic therapy trials, leading to its Food and Drug Administration (FDA) approval as the first available drug for treatment of exudative AMD ${ }^{6,7}$ While most patients receiving vPDT still lost vision, the treatment helped stabilize visual acuity and decreased lesion activity compared with sham treatment. Subsequent vPDT trials examined reduced (50\%) fluence levels in an attempt to avoid choroidal hypoperfusion and the $4 \%-6 \%$ incidence of acute vision loss associated with vPDT, and several of these trials described a trend toward better visual outcomes. ${ }^{8}$

Recently, intravitreal agents (ranibizumab and bevacizumab) have supplanted vPDT as primary therapy for neovascular AMD. These drugs bind and inactivate vascular endothelial growth factor (VEGF) and substantially improve visual acuity when delivered as repeated intravitreal injections. ${ }^{9}$ Intravitreal anti-VEGF therapy with ranibizumab (Lucentis ${ }^{\circledR}$; Genentech USA, Inc., San Francisco, CA, USA), an antihuman VEGF affinity-matured antibody fragment, was the first agent to show an increase in mean best corrected visual acuity at the 12-month time point in patients with exudative AMD. The ANCHOR (anti-VEGF antibody for the treatment of predominantly classic choroidal neovascularization [CNV] in AMD) trial demonstrated that monthly ranibizumab therapy produces significant gains in visual acuity compared with vPDT for predominantly classic subfoveal CNVM secondary to exudative macular degeneration, while MARINA (minimally classic/ occult trial of the anti-VEGF antibody ranibizumab) showed similar improvements in vision for minimally classic or occult CNVM treated with ranibizumab versus sham injections. ${ }^{10,11}$

Despite encouraging clinical results with anti-VEGF therapies (with either FDA-approved ranibizumab or the related agent, bevacizumab), most patients require repeated intravitreal injections at regular intervals to maintain initial visual acuity gains. ${ }^{12,13}$ Therapeutic approaches targeting different components of the neovascular pathophysiology in AMD have predominantly combined vPDT with anti-VEGF injections. This theoretically could decrease the need for indefinite intravitreal anti-VEGF injections while maintaining the visual acuity gains that were seen in the monthly Lucentis therapy trials. As the fluence level of light activation was empirically determined in the vPDT trials, a lower level of light fluence may be equally effective in combination with an anti-VEGF agent with less potential vPDT-related choroidal hypoperfusion damage. The LUV (Lucentis ${ }^{\circledR}$ Utilizing Visudyne $^{\circledR}$ ) study was designed to determine the threshold-dose fluence of vPDT when used in combination with intravitreal anti-VEGF therapy injections (ranibizumab), and to determine the minimum fluence dose in vPDT therapy that results in choroidal hypoperfusion.

\section{Methods}

LUV was a Phase IV comparative study evaluating the use of combination therapy with intravitreal ranibizumab and vPDT compared with intravitreal ranibizumab alone in patients with exudative AMD. In this single-site trial, approved by the Institutional Review Board, seven patients with naïve $\mathrm{CNV}$ secondary to exudative AMD were randomized at the time of entry into the study to sham vPDT (two patients), 20\% fluence vPDT (two patients), or 40\% fluence vPDT (three patients). Inclusion criteria were age $\geq 55$ years, visual acuity between 20/32 and 20/320 by ETDRS (Early Treatment Diabetic Retinopathy Study) refraction, and subfoveal neovascular membrane of any subtype confirmed by fluorescein angiography and/or indocyanine green fluorescein angiography.

Patients received standard dosages of verteporfin $\left(6 \mathrm{mg} / \mathrm{m}^{2}\right.$ body surface area) with reduced fluence (light dose of $10 \mathrm{~J} /$ $\mathrm{cm}^{2}$ at an irradiance of $120 \mathrm{~mW} / \mathrm{cm}^{2}$ for $20 \%$ fluence and $20 \mathrm{~J} / \mathrm{cm}^{2}$ at an irradiance of $240 \mathrm{~mW} / \mathrm{cm}^{2}$ for $40 \%$ fluence) delivered by diode laser over 83 seconds on day 0 of the study. On the same day, 75 minutes prior to vPDT, all patients received concomitant induction with the first of three-monthly intravitreal injections of $0.5 \mathrm{mg}$ ranibizumab.

Patients underwent ETDRS-standardized trial-frame refractions at $4 \mathrm{~m}$, clinical examination, and optical coherence tomography, at baseline and every follow-up visit. Fluorescein angiography and indocyanine green fluorescein angiography were performed at day 0 , day 1 , week 1 , and months $1,3,6$, and 9, and 12 . Retreatment with ranibizumab was given at each monthly visit (after the empiric three-monthly induction doses) if any evidence of active CNV was seen on clinical examination, qualitative-based optical coherence tomography findings, or fluorescein angiography. ${ }^{14}$ Indocyanine green fluorescein angiography choroidal hypoperfusion was graded in a masked fashion.

\section{Results}

After obtaining Institutional Review Board approval, seven patients were recruited for the study (seven males, aged 
66-80 years) and six had follow-up through to 12 months (one patient died at month 5). Despite the initial study protocol, indocyanine green fluorescein angiography could not be obtained at all intended intervals due to a national shortage of indocyanine green that began in early 2007. By the end of the study, $70 \%$ of protocol indocyanine green fluorescein angiography had been performed, and the lack of indocyanine green fluorescein angiography at certain intervals did not impact our final study results or conclusions. Median EDTRS refracted visual acuity at baseline was 20/32 in the sham group, 20/40 with $20 \%$ fluence, and 20/32 with $40 \%$ fluence (Table 1 ). One patient in the sham group had $1+$ nuclear sclerosis, one patient in the $40 \%$ fluence group had $2+$ nuclear sclerosis, and the other five patients in the study were pseudophakic; there were no other media opacities. Central retinal thickness (CRT), as measured by optical coherence tomography (Stratus III OCT; Carl Zeiss, Dublin, CA) at baseline, was 268 in the sham group, 252 in the $20 \%$ fluence group, and 298 in the $40 \%$ fluence group. By the end of the study, there were no adverse ocular or systemic safety events attributable to the use of vPDT, intravitreal ranibizumab, or their combined use.

One patient (patient 3 ) in the $20 \%$ fluence arm lost 19 letters at one year. On clinical examination, fluorescein angiogram, and indocyanine green testing, the CNVM increased markedly in size and exudation despite treatment. No other patient in any arm lost or gained $>10$ letters at one year by ETDRS refracted visual acuity (Table 1 and Figure 1). CRT decreased in every patient by the end of the study, except in patient 3 , who had a mean CRT at one year of 239, 292, and 215, respectively (Figure 2). The number of intravitreal ranibizumab treatments did not differ significantly by study group, because patients in the sham vPDT arm required an average of 7.5 injections in 12 months versus 8.0 injections in the $20 \%$ fluence vPDT group and seven injections in the $40 \%$ fluence vPDT group.

Choroidal hypoperfusion was graded in a masked fashion, and was most readily seen in the early phase of indocyanine green fluorescein angiography around the 3-5-minute time frame. No patient receiving sham vPDT had any evidence of choroidal hypoperfusion on indocyanine green angiography at baseline or at any time during follow-up (Figure 3). Patients with $20 \%$ fluence vPDT had mild vPDT-related hypoperfusion defects that appeared by week one and mostly resolved by week 4 (Figure 4), while patients with $40 \%$ fluence vPDT had marked hypoperfusion that appeared by one week and persisted even up to 12 months (Figure 5).

\section{Discussion}

vPDT was developed to treat patients with subfoveal CNV due to AMD, pathologic myopia, and ocular histoplasmosis syndrome, by reducing leakage of blood and fluid from the neovascular endothelium of the $\mathrm{CNV}^{6-8}$ Photodynamicallydamaged endothelium releases procoagulant and vasoactive factors through the lipo-oxygenase pathway (via leukotrienes) and cyclo-oxygenase pathway (via eicosanoids, such as thromboxane). These factors result in platelet aggregation, fibrin clot formation, and vasoconstriction, leading to vascular occlusion. ${ }^{15,16}$ Standard treatment involves a 10 -minute intravenous infusion of verteporfin $\left(6 \mathrm{mg} / \mathrm{m}^{2}\right.$ body surface area), followed 15 minutes after the start of the infusion by the application of $50 \mathrm{~J} / \mathrm{cm}^{2}$ light $\left(600 \mathrm{~mW} / \mathrm{cm}^{2}\right.$ for 83 seconds) to the eye.

Selectivity of verteporfin for neovascular tissues was attributed to its uptake via low-density lipoprotein (LDL) receptors and subsequent accumulation due to increased expression of LDL receptors on rapidly proliferating cells. ${ }^{17}$ Despite its relative selectivity for $\mathrm{CNV}$, standard fluence vPDT is associated with choroidal hypoperfusion that appears most intensely at one week and can persist for many months. ${ }^{18-20}$ This is seen more dramatically with indocyanine green fluorescein angiography, where the indocyanine green molecule is bound by albumin and retained mostly within the vessels, demonstrating that vPDT occludes choriocapillary vessels. Patients treated with standard fluence vPDT also

Table I Patient demographics and results of ETDRS visual acuity and central retinal thickness

\begin{tabular}{|c|c|c|c|c|c|c|c|c|c|}
\hline Patient & Age & Gender & Eye & vPDT fluence & VA (day 0) & CRT 0 & VA (1 2 months) & CRT I 2 & Injections (n) \\
\hline I & 66 & $M$ & OS & 0 & $20 / 32^{-1}$ & 269 & $20 / 32$ & 237 & 8 \\
\hline 2 & 80 & $M$ & OS & 0 & $20 / 32$ & 266 & $20 / 32$ & $24 I$ & 7 \\
\hline 3 & 77 & $M$ & OD & 0.2 & $20 / 63$ & $27 \mid$ & $20 / 160$ & 383 & II \\
\hline 4 & 79 & $M$ & OD & 0.2 & $20 / 32$ & 232 & $20 / 32$ & 201 & 5 \\
\hline 5 & 76 & $M$ & OS & 0.4 & $20 / 40^{+3}$ & 327 & $20 / 40$ & 220 & 7 \\
\hline 6 & 77 & $M$ & OD & 0.4 & $20 / 32$ & 269 & $20 / 50$ & 209 & 7 \\
\hline 7 & 77 & $M$ & OD & 0.4 & $20 / 125$ & 374 & $*$ & $*$ & 4 \\
\hline
\end{tabular}

Note: *Patient 7 died at month 5 of the study.

Abbreviations: ETDRS, Early Treatment Diabetic Retinopathy Study; VA, visual acuity; CRT, central retinal thickness; vPDT, verteporfin photodynamic therapy. 


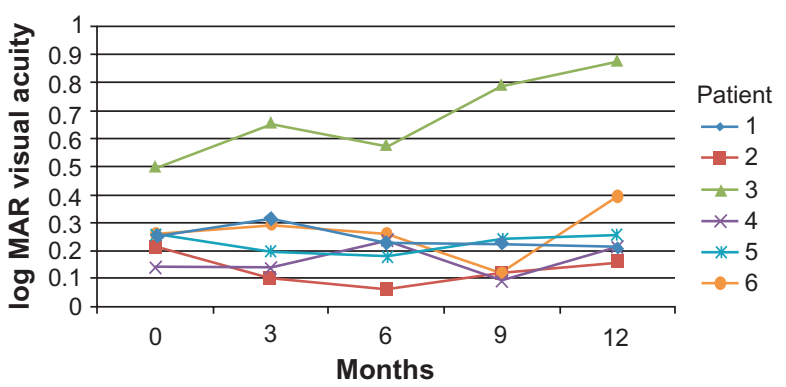

Figure I Results of logMAR BCVA over the 12 months of the study. Patient 3 (20\% vPDT fluence) lost 19 letters at one year, but no other patient in any arm lost or gained $>10$ letters at one year by ETDRS refracted visual acuity.

have an approximate $4 \%$ risk of severe vision loss in the short term after treatment, which is probably related to occlusion of blood supply to the central macula (choriocapillaris).

The rationale behind combination therapy for exudative AMD is to sustain improvements in visual acuity and angiographic outcomes with fewer treatments, by utilizing both vasoocclusive and antiangiogenic approaches. Vascular occlusion with vPDT has been shown to break down the vascular barrier, stimulating angiogenesis and VEGF expression. ${ }^{21,22}$ This VEGF expression often causes exacerbation of retinal edema immediately following vPDT treatment. Reduced fluence vPDT combined with intravitreal ranibizumab may minimize the effects of choroidal hypoperfusion and dampen the upregulated expression of VEGF and inflammatory mediators. ${ }^{22,23}$

Several reports have shown a reduced rate of potential damage to collateral structures and trends towards improved outcomes when using vPDT with half-standard fluence $(300 \mathrm{~mW} /$ $\mathrm{cm}^{2}$, a light dose of $25 \mathrm{~J} / \mathrm{cm}^{2}$ ) as monotherapy ${ }^{8}$ or in combination with intravitreal triamcinolone acetonide. ${ }^{24,25}$ Twelve-month results of the Lucentis monotherapy versus Lucentis + Visudyne in the treatment of naïve patients with Neovascular Age Related Macular Degeneration (DENALI) study recently reported that combination therapy of half-standard fluence vPDT with

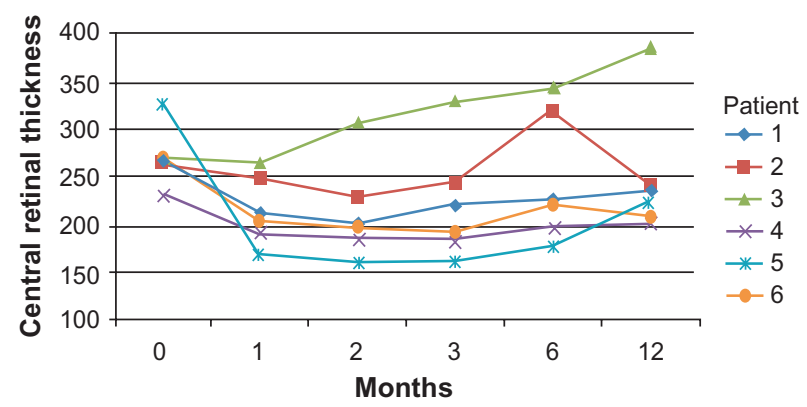

Figure 2 Results of OCT central retinal thickness over the 12 months of the study. Central retinal thickness decreased in every patient by the end of the study except in patient 3. At baseline CRT was 268 in the sham group, 252 in the $20 \%$ fluence group, and 298 in the $40 \%$ fluence group, and at one year CRT was 239, 292, and 215 respectively.
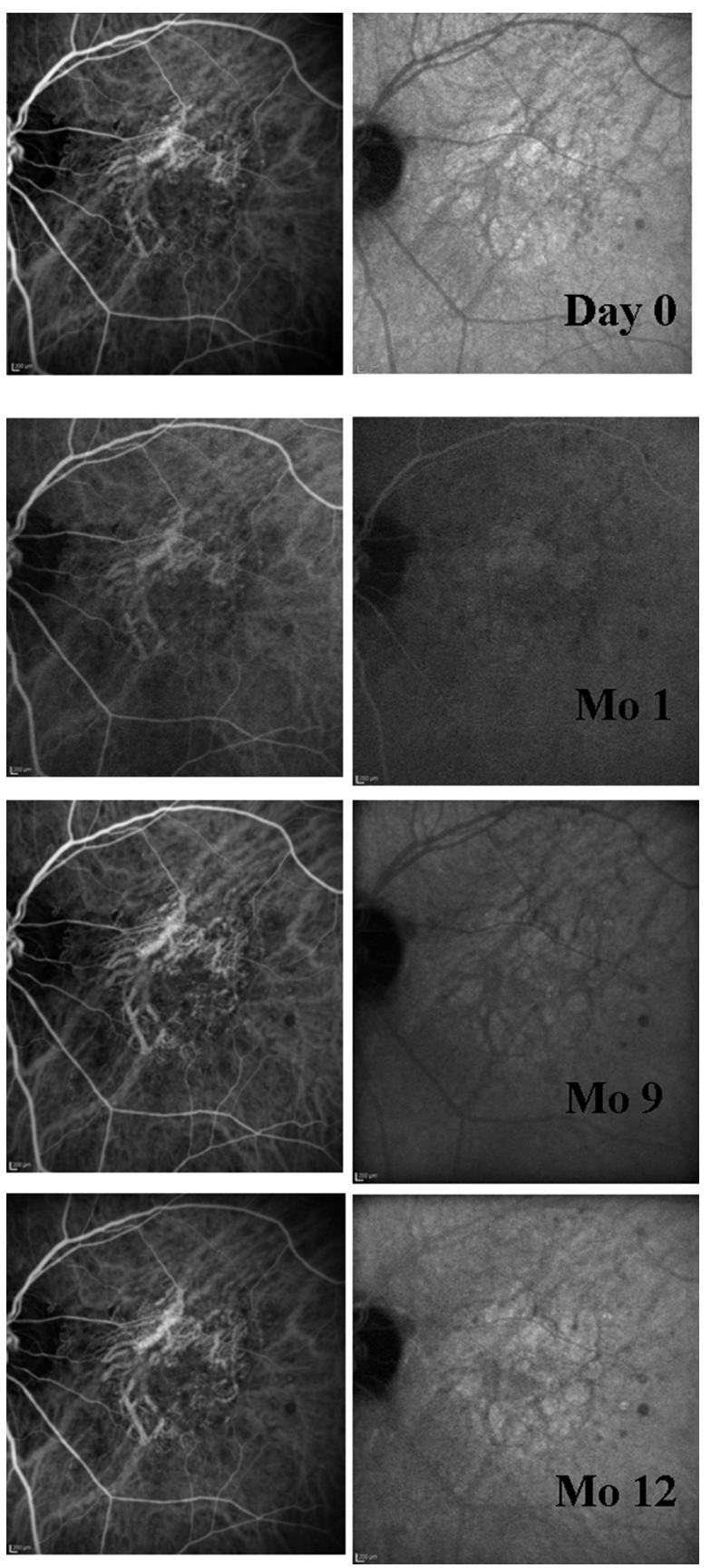

Figure 3 Early and late ICG of patient 2 ( $0 \%$ fluence vPDT). There is no evidence of choroidal hypoperfusion at any time point.

ranibizumab led to visual outcomes that were not noninferior to ranibizumab monthly monotherapy; after an initial loading dose, the combination arms also required fewer injections of ranibizumab over the remainder of the study. ${ }^{26}$ With reduced fluence, the delivery of light photons becomes the rate-determining step in the photochemical reaction. Assuming that there is more accumulation of verteporfin in the CNV because of its higher concentration of LDL receptors, even lower fluence levels of vPDT may accentuate selective treatment in the $\mathrm{CNV}$ and lessen effects in the choriocapillaries and pigment epithelial cells. 

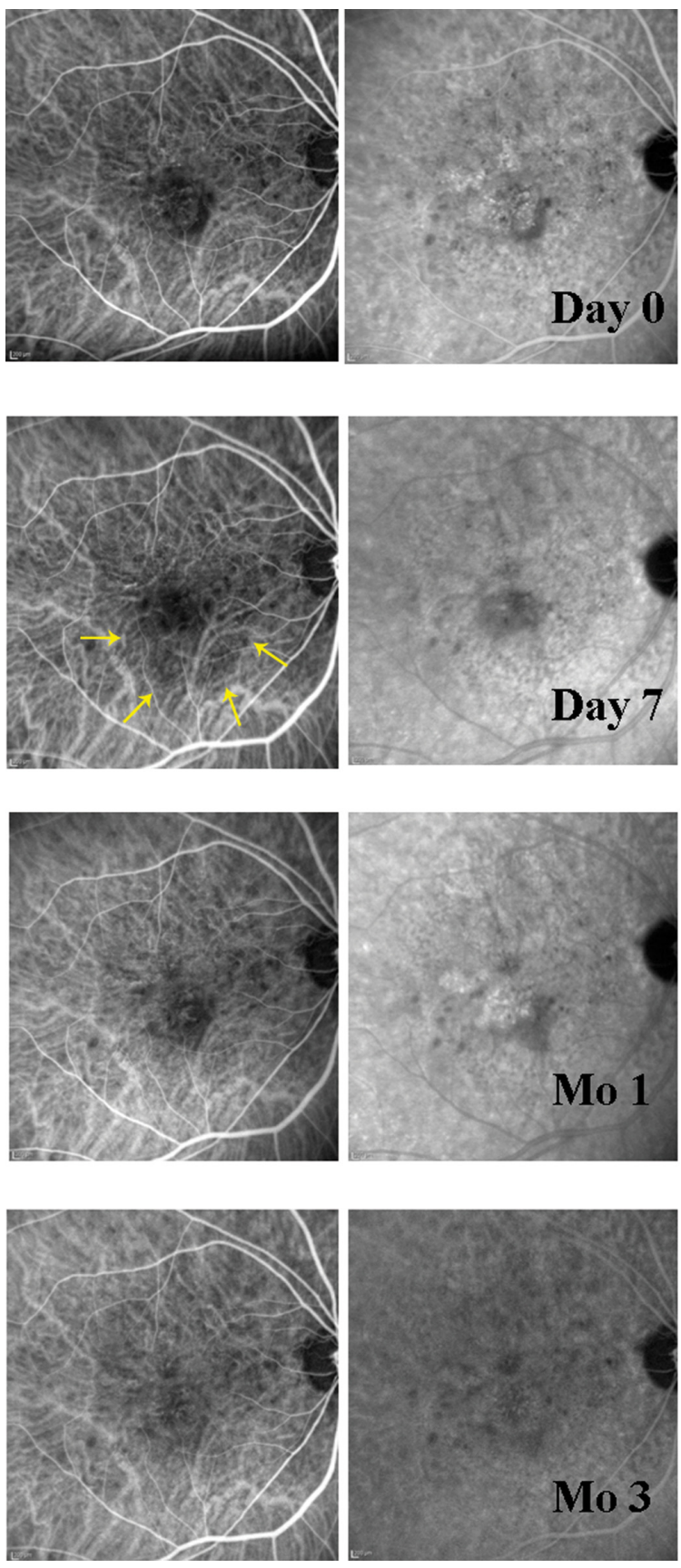

Figure 4 Early and late ICG of patient 4 (20\% fluence vPDT). By day 7 after vPDT, there is a mild but definitive area of choroidal hypoperfusion in the area of treatment (yellow arrows). By one month, this defect has resolved.

In our study, choroidal hypoperfusion related to vPDT demonstrated on indocyanine green fluorescein angiography was mild, with $20 \%$ fluence vPDT. This hypoperfusion was transient and was not demonstrable after one month and may represent the threshold-dose fluence level. The threshold dose of a drug effect is the lowest dose that demonstrates any effect on the organ or organism being treated. For most beneficial drugs, optimum effects are found at doses slightly higher than the threshold dose, while toxicity may occur at even higher levels of drug. At $40 \%$ fluence
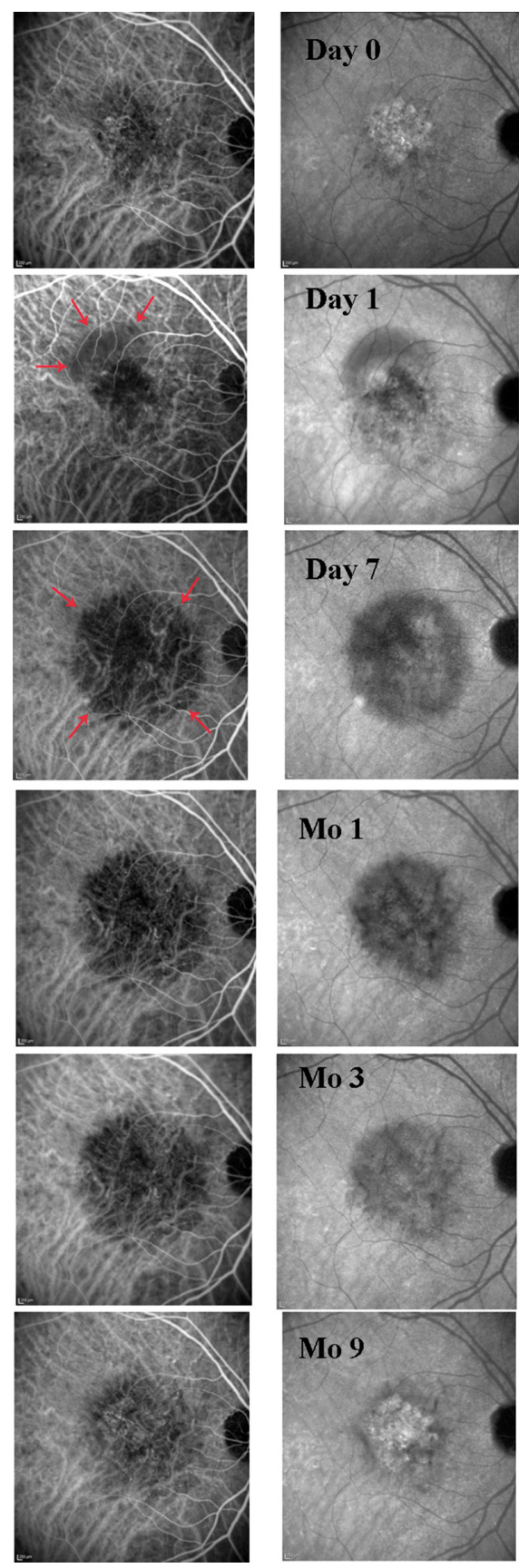

Figure 5 Early and late ICG of patient 5 (40\% fluence vPDT). As early as day I after VPDT, there is an area of superior choroidal hypoperfusion (red arrows) that becomes more pronounced by day 7 . This finding persists through month 9 of the study.

levels, choroidal hypoperfusion was significant in all patients, presenting by one week after treatment, and persisting for as long as 12 months. One patient in this group had $2+$ nuclear sclerosis, meaning that the effective fluence could be even lower than $40 \%$ fluence. Despite this evidence of a dose-related effect of vPDT on the choroidal vasculature, there did not seem to be any significant decrease in visual acuity outcomes, central retinal thickness measurements, or in the number of ranibizumab injections required when compared across the different treatment groups. 
Many studies have described choroidal hypoperfusion following vPDT, but the relationship with functional impairment remains inconclusive. A study showing minimal choroidal hypoperfusion with reduced fluence vPDT versus significant hypoperfusion with standard $\mathrm{VPDT}$ indicated the differences in hypoperfusion were not correlated with short-term clinical outcomes, and that lesion size increased even more in the reduced fluence group. ${ }^{27} \mathrm{~A}$ report of even more extensive and prolonged hypoperfusion when vPDT is combined with intravitreal ranibizumab did not see any negative effect on visual acuity, and the authors postulated that persistent inactivation of $\mathrm{CNV}$ and slow recanalization may outweigh the harm from choroidal hypoperfusion. ${ }^{20}$ Impaired perfusion may also still be compatible with good retinal function, because potential damage from oxidative radicals is limited, and neuronal or retinal tissue may be capable of increasing oxygen extraction rates under these conditions. $^{28,29}$

In the current study, hypoperfusion of the choroid was demonstrated, even with fluences much lower than what is clinically used or has been previously described. Although recruitment for the study was limited and prohibits the formation of any significant conclusions from the small number of patients, our findings imply that the vascular occlusion with vPDT may be less selective than what has been previously thought. It also suggests that this occlusion in the choriocapillaris demonstrated by indocyanine green may be necessary for effective vPDT treatment. It is encouraging that despite the choroidal hypoperfusion, there were no instances of immediate vision loss.

The LUV study examined only the lowest fluence vPDT levels, because the goal was to determine the threshold-dose fluence level of vPDT combined with intravitreal anti-VEGF therapy. It is possible that the threshold-dose fluence level of VPDT would be higher when it is not used with an agent that eliminates macular edema as effectively as ranibizumab. Because most pharmaceutical agents achieve their maximum efficacy at doses higher than the threshold dose, our pilot study suggests that a higher fluence level (more than $20 \%$ standard fluence) will probably be necessary to reduce the amount of concomitant anti-VEGF therapy. Data from the DENALI study, using 50\% fluence compared with standard fluence in combination with anti-VEGF therapy, show that a fluence level significantly above the threshold dose (but still much lower than standard fluence) may produce noninferior visual outcomes compared with monthly ranibizumab, while simultaneously requiring fewer injections. ${ }^{26}$ Further studies would be beneficial to confirm these results, because profound choroidal hypoperfusion seen at higher fluence levels may decrease the amount of anti-VEGF injections required while not compromising gains in visual acuity.

\section{Disclosure}

None of the authors have any proprietary interest in any of the data generated in this work. This study was supported in part with a research grant from Novartis. Preliminary data from this study were presented at the Association for Research in Vision and Ophthalmology annual meeting in 2009.

\section{References}

1. Friedman DS, O'Colmain BJ, Munoz B, et al. Prevalence of age-related macular degeneration in the United States. Arch Ophthalmol. 2004; 122(4):564-572.

2. Zarbin MA. Current concepts in the pathogenesis of age-related macular degeneration. Arch Ophthalmol. 2004;122(4):598-614.

3. Schmidt-Erfurth U, Hasan T, Gragoudas E, Michaud N, Flotte TJ, Birngruber R. Vascular targeting in photodynamic occlusion of subretinal vessels. Ophthalmology. 1994;101(12):1953-1961.

4. Miller JW, Walsh AW, Kramer M, et al. Photodynamic therapy of experimental choroidal neovascularization using lipoprotein-delivered benzoporphyrin. Arch Ophthalmol. 1995;113(6):810-818.

5. Kramer M, Miller JW, Michaud N, et al. Liposomal benzoporphyrin derivative verteporfin photodynamic therapy. Selective treatment of choroidal neovascularization in monkeys. Ophthalmology. 1996;103(3): 427-438.

6. Photodynamic therapy of subfoveal choroidal neovascularization in age-related macular degeneration with verteporfin: One-year results of 2 randomized clinical trials - TAP report. Treatment of age-related macular degeneration with photodynamic therapy (TAP) Study Group. Arch Ophthalmol. 1999;117(10):1329-1345.

7. Verteporfin therapy of subfoveal choroidal neovascularization in age-related macular degeneration: Two-year results of a randomized clinical trial including lesions with occult with no classic choroidal neovascularization - verteporfin in photodynamic therapy report 2 . Am J Ophthalmol. 2001;131(5):541-560.

8. Azab M, Boyer DS, Bressler NM, et al. Verteporfin therapy of subfoveal minimally classic choroidal neovascularization in age-related macular degeneration: 2-year results of a randomized clinical trial. Arch Ophthalmol. 2005;123(4):448-457.

9. Ferrara N, Davis-Smyth T. The biology of vascular endothelial growth factor. Endocr Rev. 1997;18(1):4-25.

10. Brown DM, Kaiser PK, Michels M, et al. Ranibizumab versus verteporfin for neovascular age-related macular degeneration. $N$ Engl J Med. 2006;355(14):1432-1444.

11. Rosenfeld PJ, Brown DM, Heier JS, et al. Ranibizumab for neovascular age-related macular degeneration. $N$ Engl J Med. 2006;355(14):1419-1431

12. Regillo CD, Brown DM, Abraham P, et al. Randomized, doublemasked, sham-controlled trial of ranibizumab for neovascular agerelated macular degeneration: PIER Study year 1. Am J Ophthalmol. 2008;145(2):239-248.

13. Lalwani GA, Rosenfeld PJ, Fung AE, et al. A variable-dosing regimen with intravitreal ranibizumab for neovascular age-related macular degeneration: Year 2 of the PRONTO study. Am J Ophthalmol. 2009; 148(1):43-58.e1.

14. Brown DM, Regillo CD. Anti-VEGF agents in the treatment of neovascular age-related macular degeneration: Applying clinical trial results to the treatment of everyday patients. Am J Ophthalmol. 2007; 144(4):627-637.

15. Fingar VH, Wieman TJ, Doak KW. Role of thromboxane and prostacyclin release on photodynamic therapy-induced tumor destruction. Cancer Res. 1990;50(9):2599-2603. 
16. Schmidt-Erfurth U, Hasan T. Mechanisms of action of photodynamic therapy with verteporfin for the treatment of age-related macular degeneration. Surv Ophthalmol. 2000;45(3):195-214.

17. Allison BA, Pritchard PH, Levy JG. Evidence for low-density lipoprotein receptor-mediated uptake of benzoporphyrin derivative. Br J Cancer: 1994;69(5):833-839.

18. Gelisken F, Lafaut BA, Inhoffen W, Voelker M, Grisanti S, Bartz-Schmidt KU. Clinicopathological findings of choroidal neovascularisation following verteporfin photodynamic therapy. $\mathrm{Br} J$ Ophthalmol. 2004;88(2):207-211.

19. Schlotzer-Schrehardt U, Viestenz A, Naumann GO, Laqua H, Michels S, Schmidt-Erfurth U. Dose-related structural effects of photodynamic therapy on choroidal and retinal structures of human eyes. Graefes Arch Clin Exp Ophthalmol. 2002;240(9):748-757.

20. Kiss CG, Wagner J, Simader C, Michels S, Schmidt-Erfurth U. Effect of photodynamic therapy (PDT) and ranibizumab on vascular anatomy and retinal function in neovascular age-related macular degeneration. [abstract] Invest Ophthalmol Vis Sci. 2007;48:4544.

21. Michels S, Schmidt-Erfurth U. Sequence of early vascular events after photodynamic therapy. Invest Ophthalmol Vis Sci. 2003;44(5): 2147-2154.

22. Schmidt-Erfurth U, Schlotzer-Schrehard U, Cursiefen C, Michels S, Beckendorf A, Naumann GO. Influence of photodynamic therapy on expression of vascular endothelial growth factor (VEGF), VEGF receptor 3, and pigment epithelium-derived factor. Invest Ophthalmol Vis Sci. 2003;44(10):4473-4480.
23. Tatar O, Adam A, Shinoda K, et al. Influence of verteporfin photodynamic therapy on inflammation in human choroidal neovascular membranes secondary to age-related macular degeneration. Retina. 2007;27(6):713-723.

24. Singh CN, Saperstein DA. Combination treatment with reduced-fluence photodynamic therapy and intravitreal injection of triamcinolone for subfoveal choroidal neovascularization in macular degeneration. Retina. 2008;28(6):789-793.

25. Sacu S, Varga A, Michels S, et al. Reduced fluence versus standard photodynamic therapy in combination with intravitreal triamcinolone: Short-term results of a randomised study. Br J Ophthalmol. 2008; 92(10):1347-1351.

26. DENALI Study Group. Efficacy and safety of verteporfin PDT in combination with ranibizumab compared to ranibizumab monotherapy in AMD: 12-month outcome of the DENALI study. Presented at World Ophthalmology Congress. Jun 5-9, 2010, Berlin, Germany.

27. Michels S, Hansmann F, Geitzenauer W, Schmidt-Erfurth U. Influence of treatment parameters on selectivity of verteporfin therapy. Invest Ophthalmol Vis Sci. 2006;47(1):371-376.

28. Wise RJ, Bernardi S, Frackowiak RS, Legg NJ, Jones T. Serial observations on the pathophysiology of acute stroke. The transition from ischaemia to infarction as reflected in regional oxygen extraction. Brain. 1983;106(Pt 1):197-222.

29. Schmidt-Erfurth U, Kiss C, Sacu S. The role of choroidal hypoperfusion associated with photodynamic therapy in neovascular age-related macular degeneration and the consequences for combination strategies. Prog Retin Eye Res. 2009;28(2):145-154.
Clinical Ophthalmology

\section{Publish your work in this journal}

Clinical Ophthalmology is an international, peer-reviewed journal covering all subspecialties within ophthalmology. Key topics include: Optometry; Visual science; Pharmacology and drug therapy in eye diseases; Basic Sciences; Primary and Secondary eye care; Patien Safety and Quality of Care Improvements. This journal is indexed on

Submit your manuscript here: http://www.dovepress.com/clinical-ophthalmology-journal

\section{Dovepress}

PubMed Central and CAS, and is the official journal of The Society of Clinical Ophthalmology (SCO). The manuscript management system is completely online and includes a very quick and fair peer-review system, which is all easy to use. Visit http://www.dovepress.com/ testimonials.php to read real quotes from published authors. 